\title{
Pertussis
}

\section{Experten empfehlen Auffrischimpfung alle zehn Jahre}

- Dank erfolgreicher Impfprogramme ist Pertussis bei jungen Kindern gut kontrolliert - nicht aber bei Erwachsenen. „Denn der Immunschutz bei Keuchhusten ist nicht lebenslang", erklärte Prof. Fred Zepp, Mainz. Nach Impfung hält er vier bis zwölf Jahre an, nach durchgemachter Infektion bis zu 20 Jahre. Ab dem achten Jahr nach der letzten Auffrischimpfung - und vorangegangener Grundimmunisierung mit vier Impfungen bis zum 14. Lebensmonat - steigt das Infektionsrisiko auf $20-32 \%$.

Die nachlassende Immunität macht Adoleszenten und Erwachsene wieder empfindlich für Bordetella pertussis und trägt wesentlich zu der ansteigenden Inzidenz in dieser Altersgruppe bei. Weil Keuchhusten infolge Teilimmunität nach Impfung bzw. Infektion dann meist untypisch verläuft, wird er häufig als chronischer Husten fehldiagnostiziert. Mit fatalen Folgen: Heranwachsende und Erwachsene sind die Hauptinfektionsquelle für Neugeborene und Säuglinge, die noch nicht oder erst partiell immunisiert sind. Sie

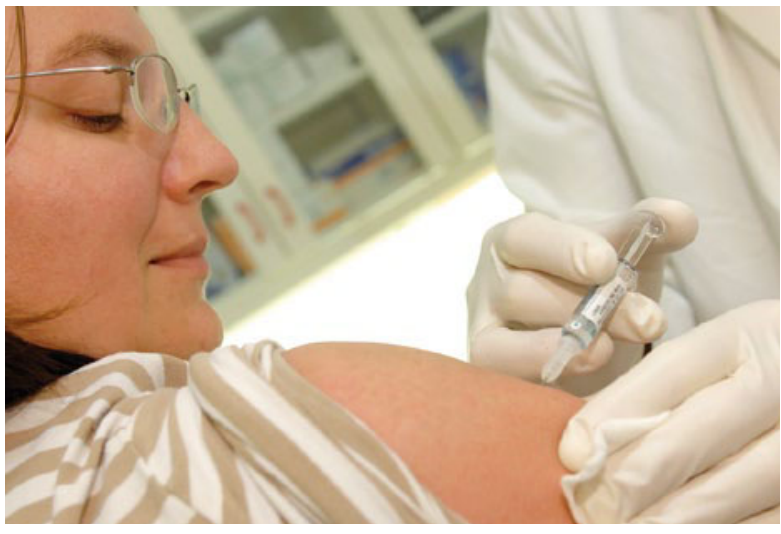

Ausreichende Immunität gegen Bordetella pertussis: Schützt nicht nur den Erwachsenen, sondern auch Neugeborene. nicht nur bei Säuglingen, Kleinkindern und im Vorschulalter, wie bereits in den meisten Ländern Europas etabliert, sondern auch in der Adoleszenz und bei Erwachsenen, forderte Zepp. Dafür stünden wirksame und gut verträgliche Vakzinen zur Verfügung. Die Experten-Gruppe COPE (Consensus on pertussis booster vaccination in Europe) empfiehlt, die existierende Diphtherie-Tetanus-Auffrischimpfung für Heranwachsende und Erwachsene um Pertussis zu ergänzen (also dTpa statt nur dT) mit anschließender Boosterung haben das höchste Risiko für Komplikationen: Zwei Drittel der Kinder mit Pertussis im ersten Lebensjahr müssen für Wochen ins Krankenhaus, jedes fünfte Kind entwickelt eine Pneumonie, bei über $2 \%$ kommt es zu Krampfanfällen, mehr als $1 \%$ stirbt.

\section{„Tödliches Muster der}

\section{Pertussis-Übertragung"}

Um dieses "tödliche Muster der PertussisÜbertragung" zu unterbrechen, ist ein lebenslanges Impfprogramm erforderlich alle zehn Jahre. Solange eine suffiziente Boosterung nicht etabliert ist, sei darüber hinaus eine Cocooning-Strategie zum Schutz der Ungeschützten anzustreben, bei der Eltern und Geschwister als nahe Kontakte Neugeborener, die noch zu jung für die Vakzine sind, geimpft werden.

\footnotetext{
- Michael Koczorek

Quelle: Satelliten-Symposium auf der Jahrestagung der European Society for Pediatric Infectious Diseases (ESPID), Den Haag, Juni 2011 (Veranstalter: GlaxoSmithKline)
}

\section{Selbstmedikation bei Rücken-, Muskel- und Gelenkschmerzen Das sagen die Patienten}

— Effizienz, Verträglichkeit und Akzeptanz sind die wesentlichen Parameter, wenn es um eine topische Behandlung von Rücken-, Muskel- und Gelenkschmerzen geht. Wie es speziell in der Selbstmedikation um diese Parameter bestellt ist, untersuchte eine Beobachtungsstudie zu doc ${ }^{\circledast}$ Ibuprofen Schmerzgel.

64 Apotheken im gesamten Bundesgebiet waren eingebunden. Von Oktober 2010 bis Februar 2011 wurden die Daten von insgesamt 576 Patienten (Durchschnittsalter 54 Jahre) mit Rücken-, Muskel- und Gelenkschmerzen erhoben. Die topische Schmerzbehandlung sollte - in Abhängigkeit vom Verlauf - bis zu zehn Ta- ge je dreimal täglich erfolgen. Tatsächlich verwendeten die Studienteilnehmer das Gel durchschnittlich 2,6-mal täglich über einen Zeitraum von 7,4 Tagen - was für eine gute Compliance nach der Beratung durch den Apotheker spricht. Bei einer allgemeinen Bewertung sagten den Patienten vor allem das "rasche Einziehen in die Haut" (68\%), die "gute Verteilbarkeit auf der Haut" (56\%) und der „angenehme Geruch" (51\%) zu.

In $84 \%$ der Fälle waren die Beschwerden bereits nach etwas mehr als sieben Behandlungstagen viel besser (31\%) oder besser (53\%). Im Durchschnitt stellte sich nach etwas mehr als fünf Tagen eine voll- ständige Beschwerdefreiheit ein. „Besonders gefallen" hatte den oft von chronischen oder starken Schmerzen geplagten Studienteilnehmern die "schnelle Schmerzbefreiung" (52\%). Diese Eigenschaft wird auf die besondere Galenik von $\mathrm{doc}^{\circledast}$ Ibuprofen Schmerzgel zurückgeführt: Da der Wirkstoff in nanometerkleinen Mizellen vollständig gelöst vorliegt, kann das Gel die Haut rückstandsfrei penetrieren. Daher erfolgt der analgetische Wirkeintritt besonders schnell. Auch die Verträglichkeit wurde von den Teilnehmern günstig beurteilt.

\footnotetext{
- Red.

Nach Angaben von Hermes
} 